Nationales Versorgungszentrum Schmerz

\title{
Schmerztherapie muss besser werden
}

_ „Die Qualität der schmerzmedizinischen Versorgung in Deutschland leidet an unzureichenden Strukturen und Defiziten in der Ausbildung von Ärzten“, konstatieren die Deutsche Gesellschaft für Schmerzmedizin und die Selbsthilfeorganisation Deutsche Schmerzliga. Leidtragende sind die Patienten.

„Verantwortlich für die Versorgungsdefizite ist vor allem", so der Präsident der Deutschen Schmerzliga, PD Dr. Michael Überall, Nürnberg, ,die an Fachgebieten und ihren Grenzen orientierte Ausbildung der Ärzte, die den Bedürfnissen von Patienten mit chronischen Schmerzen nicht gerecht wird.“

Die chronische Schmerzkrankheit umfasst den ganzen Menschen mit kör- perlichen, psychischen sowie sozialen Aspekten und verlangt umfassende neurologische, psychologische, funktionell-orthopädische und anästhesiologische Kenntnisse. Derzeit gibt es in Deutschland 23 Millionen Schmerzpatienten. Jeder Zehnte von ihnen leidet unter einer schweren Schmerzerkrankung.

Notwendig seien daher eine strukturierte Ausbildung in der Schmerzmedizin, die Bedarfsplanung für die ambulante und stationäre schmerzmedizinische Versorgung sowie die Einführung des Facharztes für Schmerzmedizin. -

Red.

- Nach Informationen der Deutschen Gesellschaft für Schmerzmedizin

\section{Kurz notiert}

Europäische Kommission genehmigt ungeboostertes Atazanavir bei Ritonavir-Unverträglichkeit $\rightarrow$ Atazanavir (Reyataz ${ }^{\circledast}$ ) kann ab sofort auch ohne den Booster Ritonavir eingesetzt werden. Damit können HIV-Patienten mit Unverträglichkeit gegenüber Ritonavir eine Therapie mit $400 \mathrm{mg}$ Atazanavir ohne Booster fortführen, wenn sie mindestens schon sechs Monate unter der virologischen Nachweisgrenze $(<50 / \mathrm{ml})$ sowie immunologisch stabil sind. Die ungeboosterte Anwendung wird nicht empfohlen bei Patienten mit vorherigem virologischem Therapieversagen, nachgewiesenen Resistenzmutationen, erwarteten Complianceschwierigkeiten, gleichzeitiger Anwendung von Medikamenten, die die Bioverfügbarkeit von Atazanavir reduzieren (Tenofovir, Protonenpumpeninhibitoren, $\mathrm{H}_{2}$-Rezeptorantagonisten) sowie bei Schwangeren.

Red.

\section{Informations- und Service-Website für Diabetesfachkräft}

\section{Mit einem Click auf dem neuesten Stand}

_ Um kaum eine andere Erkrankung ranken sich so viele Mythen, und selten gibt es so viele Vorurteile wie beim Diabetes mellitus. Der News-Bereich der Website www.msd-diabetes.de sorgt durch Verweis auf aktuelle Neuigkeiten für Aufklärung. Im Bereich „Wissen“ gibt es viele Informationen und Tipps rund um das Thema Diabetes. Hinzu kommt ein kleines Faltblatt für Men-

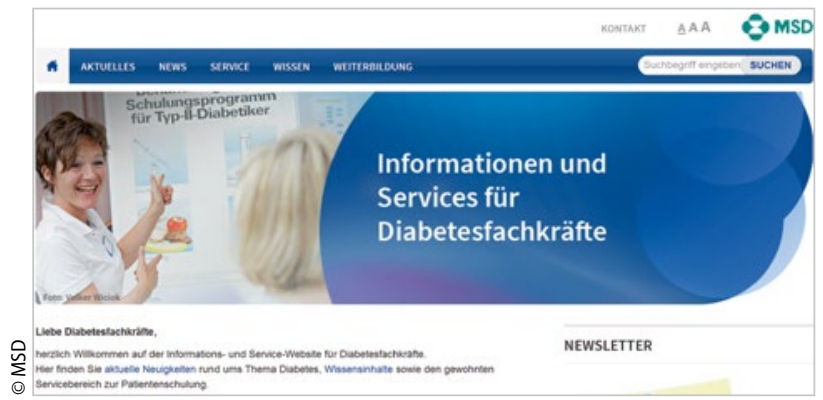

schen, die keinen Diabetes haben, das die Kommunikation mit Diabetespatienten im privaten Umfeld fördern soll.

\section{Umfangreiche Materialien}

Im Bereich „Service“ stehen Materialien zur Schulung von Typ-2-Diabetikern bereit: die Broschüre „Leben mit Diabetes“ mit Antworten von Experten sowie Tipps und Tricks und die Informationsbroschüre „Dranbleiben am Cholesterin“ mit vielen Ratschlägen für den Alltag. Quizblöcke, beispielsweise zu "Zuckerfallen“ und versteckten Fetten, bieten die Möglichkeit, das Wissen rund um das Thema Diabetes auf kurzweilige Art zu vertiefen. Damit Patienten mit Diabetes wichtige Informationen stets griffbereit haben, werden ein Notfallausweis und ein Gesundheitspass zur Dokumentation von Krankheitsverlauf und Therapieempfehlungen angeboten.

\section{Kostenloser Newsletter}

Um stets auf dem aktuellen Stand zu sein, gibt es für Diabetesfachkräfte auch die Möglichkeit, einen kostenlosen Newsletter zu abonnieren, der aktuell über Themen rund um Diabetes und kardiovaskuläre Erkrankungen informiert und praktische Tipps zu Ernährung und Bewegung gibt. Daneben finden sich immer wieder interessante Hinweise zur eigenen Fortbildung und fachliche Ausblicke.

- Red.

- Nach Informationen von MSD 